\section{Continence rates should be interpreted with caution in patients with overactive bladder}

Urge urinary incontinence (UUI) is a symptom of overactive bladder. Antimuscarinic agents are the first-line therapy for patients with overactive bladder, and the continence rate (the percentage of patients who record no urinary incontinence episodes during a diary period) is often used as a measure of treatment success. Wein and colleagues examined whether continence rates truly reflect the efficacy of antimuscarinic treatment.

The authors performed a post-hoc analysis of data from a 12-week, multinational, randomized, double-blind trial. Participants (mean age 61 years) with urinary frequency ( $\geq 8$ voids per $24 \mathrm{~h}$ ) and UUI ( $\geq 5$ episodes per week) were randomly allocated to receive $4 \mathrm{mg}$ extendedrelease tolterodine or placebo once daily. The authors conducted intention-to-treat and perprotocol analyses of weekly UUI episodes at baseline and week 12. Patients kept voiding diaries, and these data were analyzed for the 3,5 , and 7 -day periods immediately preceding both assessments.

In the intention-to-treat and per-protocol analyses 503 and 374 patients received placebo and 502 and 398 patients received tolterodine, respectively. The overall continence rate decreased with increasing number of UUI episodes at baseline and increased diary duration. Higher continence rates were observed in the per-protocol analysis than in the intention-to-treat analysis.

The authors conclude that diary duration, baseline frequency of UUI and the patient population analyzed all influence continence rateswhich, therefore, might not be a valid measure of efficacy in trials of antimuscarinic drugs, unless standard methodology and a placebo-controlled design are used.

Original article Wein AJ et al. (2007) Achieving continence with antimuscarinic therapy for overactive bladder: effects of baseline incontinence severity and bladder diary duration. BJU Int 99: 360-363

\section{Percutaneous renal core biopsy results should influence treatment of patients}

The accuracy and reliability of percutaneous renal biopsy has been much debated.
Tissue-based diagnostic assessment remains important, because although CT, ultrasonography and MRI can accurately differentiate between solid and cystic lesions, they have limited ability to distinguish benign from malignant masses. Maturen and colleagues, therefore, evaluated the accuracy of imaging-guided percutaneous needle core biopsy of renal masses, and determined its effect on patients' clinical management.

The authors retrospectively reviewed medical records and biopsy data on 152 renal masses from 125 patients (age range 28-90 years, 70 male). All biopsies were performed under CT or ultrasonographic guidance and used a coaxial $18 \mathrm{G}$ core needle.

Malignant neoplasm was found in $56 \%$ of biopsies, benign findings in $40 \%$, and $4 \%$ were nondiagnostic. Two patients experienced postprocedural hematomas. One patient experienced retroperitoneal bleeding from a ruptured pseudoaneurysm 3 months after surgery. No other complications, especially needle-track tumor seeding, were observed. The sensitivity and specificity of percutaneous renal mass biopsy to detect malignancy were $97.7 \%$ and $100 \%$, respectively. Biopsy diagnostic accuracy was not affected by the method of imaging guidance. Approximately $60 \%$ of the biopsy results profoundly changed the patients' clinical management (i.e. a change between therapy and no therapy). Altered management was particularly beneficial for those who avoided unnecessary nephrectomy because of their biopsy results.

The authors conclude that percutaneous renal mass core biopsy is a highly sensitive, specific and safe method of characterizing renal masses, the results of which should be considered in patients' clinical management.

\section{Original article Maturen KE et al. (2007) Renal mass core biopsy: accuracy and impact on clinical management. AJR Am J Roentgenol 188: 563-570 \\ Intracavernosal injection results in accurate measurement of penile curvature}

The decision whether to treat Peyronie's disease medically or with a surgical approach depends on disease duration and the extent of fibrous plaque formation within the penile tunica albuginea-the cause of pain and curvature during erections. Disease severity is assessed by measurement of the degree of curvature of the fully erect 